\section{Perfil do aleitamento materno em menores de um ano no Município de Londrina, Paraná}

\section{Breastfeeding profile in babies below one year old in the municipality of Londrina, State of Parana, Brazil}

Marli T. O. Vannuchi 1

Zuleika Thomson 2

Maria M. L. Escuder 3

Mauren T. G. M. Tacla 4

Kátia M. K. Vezozzo 5

Lilian M. C. P. de Castro 6

Márcia M. B. de Oliveira 7

Sonia I. Venancio 8

1,4 Departamento de Enfermagem Universidade Estadual de Londrina. Rua Weyner J. Maciel Alves, 179. Londrina, PR, Brasil. CEP: 86.055-500 E-mail: mtacla@sercomtel.com.br

2 Departamento de Pediatria e Cirurgia Pediátrica. Universidade Estadual de Londrina, PR, Brasil.

${ }^{3}$ Instituto de Saúde de São Paulo, SP, Brasil.

5 Curso de Enfermagem. Centro Universitário Filadélfia. Londrina, PR, Brasil.

${ }^{6}$ Secretaria Municipal de Saúde de Londrina. Coordenadoria do Comitê de Estímulo ao Aleitamento Materno. Londrina, PR, Brasil.

7 Departamento de Enfermagem. Universidade Estadual de Londrina, PR, Brasil.

8 Instituto de Saúde de São Paulo, SP, Brasil.

\section{Abstract}

Objectives: to describe and analyze data of a survey on nutritional practices in children to one year of age carried out in Londrina, Paraná

Method: data were obtained during the "National Immunisition Canpaign", carried out in August 2002. Mothers were asked to answer a questionnaire on how they had fed their child in the preceding 24 hours. Children were selected following a sample planning by cluster. Probits analysis was used to know the breastfeeding frequency. Through logistic regression analysis risk factors were analyzed concerning the interruption of exclusive breastfeeding of babies younger than four months and the weaning of babies below the age of one.

Results: among babies younger than four months exclusive breastfeeding (EB) prevalence was $29.3 \%$ and predominant breastfeeding (PB) was 20.7\%. For those younger than six months, EB prevalence was $21 \%$ and $P B$ was $16.5 \%$. Concerning children below the age of one, the prevalence was $62.4 \%$. The median time was 11.9 days for $E B$ and 257.3 for PB. Risk factors were: use of pacifiers, first child, mother's age and job.

Conclusions: the breast-feeding rates found were considered low, showing the necessity of making adequate public policies.

Key words Breast feeding, Weaning, Lactation, Nutrition, survey

\section{Resumo}

Objetivos: descrever e analisar dados de um inquérito realizado em Londrina, Paraná, sobre práticas alimentares no primeiro ano de vida.

Métodos: os dados foram obtidos durante a "Campanha Nacional de Vacinação, realizada em agosto de 2002. As crianças foram selecionadas por plano de amostragem por conglomerado. Aplicou-se um questionário com questões sobre alimentação da criança nas 24 horas precedentes. Para obtenção das freqüencias empregou-se análise de probitos. Pela análise de regressão logística, foram analisados os fatores de risco para interrupção da amamentação exclusiva em menores de quatro meses e desmame em menores de um ano.

Resultados: em menores de quatro meses, a prevalência do aleitamento materno exclusivo (AME) foi $29,3 \%$ e, para aleitamento materno predominante (AMP) 20,7\%. Nos menores de seis meses, a prevalência do AME foi 21,0\% e do AMP 16,5\%. Para o aleitamento materno (AM) em menores de um ano, a prevalência foi $62,4 \%$. As medianas em dias foram 11,9 para AME e 257,3 para AM. Entre os fatores de risco para inter rupção do AME e do AM destacam-se: uso de chupeta, primiparidade, acompanhamento ambulato rial em serviço público, idade e trabalho materno.

Conclusões: as taxas de aleitamento foram consideradas baixas, indicando a necessidade de se estabelecerem políticas públicas adequadas para alimentação da criança.

Palavras-chave Aleitamento materno, Desmame, Lactação, Inquéritos nutricionais 


\section{Introdução}

O leite materno apresenta, em sua composição, todos os nutrientes necessários à criança nos seis primeiros meses de vida. Além de ser facilmente digerido e assimilado pelo lactente, é livre de impurezas e está sempre à temperatura ideal; muito mais econômico do que as fórmulas industrializadas, promove o melhor desenvolvimento físico e mental além de conferir proteção contra diarréias e mortes por doenças infecciosas. Em virtude de todos os benefícios, a World Health Organization (WHO) ${ }^{1}$ recomenda que as crianças sejam amamentadas exclusivamente ao seio, sem alimentos complementares até o sexto mês de vida. Após essa idade, é necessária a introdução de outros alimentos à dieta infantil, mantendo-se o aleitamento materno até 24 meses ou mais.

Há três décadas o município de Londrina vem desenvolvendo ações em favor da amamentação. Em 1994, foi criado o Comitê de Estímulo ao Aleitamento Materno (CALMA) do município de Londrina pela Secretaria Municipal de Saúde, com a finalidade de integrar e avaliar ações de promoção, proteção e apoio ao aleitamento materno no município, congregando todas as instituições que trabalham com o tema. ${ }^{2}$ Esse comitê vem colaborando com os planejadores das ações de saúde do município, fornecendo indicadores confiáveis da prática de alimentação infantil, com vistas ao desenho e avaliação de intervenções apropriadas.

Nesse sentido, no final de 2001, com a inexistência de dados precisos e atualizados sobre a situação do aleitamento materno em Londrina, a exemplo dos estudos realizados por Venancio et al.,3 Venancio 4 e Audi et al.,5 o CALMA instrumentalizou-se para fazer um levantamento epidemiológico realizado em campanhas de vacinação, possibilitando um diagnóstico rápido da situação da amamentação e das práticas de desmame, com um baixo custo. É importante destacar que a $\mathrm{WHO}^{1,8}$ propôs indicadores para o acompanhamento da situação do aleitamento para que seja atingida a meta de $100 \%$ das crianças em Amamentação Exclusiva (AME) até os seis meses de idade.

Este artigo teve como objetivo descreve e analisar os resultados do perfil do aleitamento materno no município de Londrina, a partir dos dados coletados em inquérito sobre práticas alimentares no primeiro ano de vida, no "Dia Nacional de Campanha de Vacinação" no Município de Londrina.

\section{Métodos}

Londrina é um município localizado ao norte do Estado do Paraná, Brasil e tem uma população estimada pelo Instituto Brasileiro de Geografia e Estatística (IBGE), 6 em 2002, de 460.910 habitantes, dos quais 7654 são menores de um ano. Visando contemplar esse segmento, foi aplicado o inquérito "Práticas Alimentares no Primeiro Ano Vida", que faz parte do "Projeto Amamentação e Municípios" do Instituto de Saúde da Secretaria de Estado da Saúde de São Paulo. ${ }^{3}$ Com a finalidade de padronizar as condutas no campo de pesquisa, as pesquisadoras responsáveis pela coordenação do estudo no município receberam treinamento de oito horas no Instituto de Saúde de São Paulo, tendo sido orientadas sobre: metodologia de pesquisa, amostragem, coleta de dados, capacitação de supervisores de campo e entrevistadores. Trata-se de um estudo do tipo transversal realizado nas crianças menores de um ano, que foram aos postos de vacinação durante a "Campanha Nacional de Vacinação" realizada em agosto de 2002. O "Projeto Amamentação e Municípios" propõe o uso desse espaço para rea-lização de inquéritos de amamentação desde 1998, com experiências nos estados de São Paulo, Paraíba e Rio de Janeiro. Para permitir a inferência dos dados coletados, é necessário que a cobertura vacinal na campanha no município seja igual ou superior a $85 \%$.

$\mathrm{O}$ inquérito em Londrina foi realizado aplicandose uma amostra por conglomerados, representados pelos postos de saúde. Planejou-se um tamanho de amostra de 2000 crianças menores de um ano, com a finalidade de se calcular a mediana do aleitamento materno exclusivo usando análise de probitos e obter os indicadores de aleitamento materno em diversas faixas etárias.

Como os postos de vacinação eram de diferentes tamanhos, o sorteio foi realizado em duas etapas, com probabilidade proporcional ao tamanho dos conglomerados (PPT). Segundo Silva, ${ }^{7}$ esse processo mantém a amostra autoponderada, pois permanece constante a expressão $f=f_{1} \cdot f_{2}$, onde $f_{1}$ é componente da primeira etapa do sorteio e $f_{2}$, o componente da segunda etapa. Na primeira etapa sortearam-se os postos de vacinação, de forma sistemática, usando como base o número de crianças vacinadas em cada posto de vacinação na campanha anterior. A relação dos postos foi ordenada segundo região da cidade, de modo que o 
sorteio respeitou a proporcionalidade populacional de cada região. A segunda etapa realizou-se na fila de vacinação. Cada posto teve sua fração de sorteio $\mathrm{f}_{2}$, segundo a qual os entrevistadores foram orientados para conduzir o sorteio sistemático na fila de crianças. Desse modo, os postos maiores tiveram maior probabilidade de sorteio no primeiro estágio $\left(f_{1}\right)$, e as crianças dos postos menores tiveram maior probabilidade de sorteio no segundo estágio $\left(\mathrm{f}_{2}\right)$. Utilizou-se o estimador deff (efeito de delineamento) como estratégia de validação da técnica amostral, e o intervalo de confiança (IC95\%) para análise da precisão da amostra. ${ }^{7}$

Dos 152 postos programados para a campanha de 2002, foram sorteados 39. Para cada posto estimou-se 50 entrevistas e um dos postos foi sorteado duas vezes, devido ao grande número de crianças previstas.

A coleta de dados foi realizada por meio de uma entrevista aplicada às mães ou acompanhantes de crianças menores de um ano, que procuraram os postos de vacinação sorteados para a pesquisa, na segunda etapa da "Campanha de Vacinação", em agosto de 2002. Esse questionário é composto por questões sobre alimentação da criança nas últimas 24 horas além de questões sobre os serviços de saúde e características das mães e das crianças. Foi aplicado por estudantes universitários de cursos da área da saúde, previamente treinados e supervisionados durante o processo de coleta de dados, confome relatórios com indicadores de aleitamento materno baseados nas definições propostas pela $\mathrm{WHO} .8$

$\mathrm{O}$ aleitamento Materno Exclusivo (AME): foi definido como aquele em que a criança recebe apenas leite materno de sua mãe ou ama-de-leite, ou leite materno ordenhado, e não recebe outros líquidos ou sólidos com exceção de vitaminas, suplementos minerais ou medicamentos.

Enquanto que o aleitamento Materno Predominante (AMP) foi considerado como aquele em que a fonte predominante de nutrição da criança é o leite materno. Porém, a criança também pode receber água e bebidas à base de água (água açucarada e com sabores, infusões, chá, etc), suco de frutas, solução de sais de hidratação oral (SRO), vitaminas, minerais e medicamentos em gotas ou xaropes, e líquidos cerimoniais (em quantidades limitadas).

O Aleitamento Materno (AM) foi definido como aquele em que a criança recebe leite materno diretamente do peito ou ordenhado, independentemente de receber outros alimentos.

Empregou-se a análise de probitos ${ }^{9}$ para a obtenção da mediana da amamentação expressa em dias. O modelo de probitos permite estimar as freqüências em pontos exatos a partir de regressões lineares ponderadas, considerando a amostra total de crianças do estudo. Assim, os resultados recebem menor influência das flutuações amostrais que ocorrem mês a mês. Essa análise oferece os valores preditivos de crianças em desmame através de uma regressão linear ponderada de probitos (y) em relação a (x), utilizando o procedimento da veromaximos semelhança. A relação entre probitos (y) e valores de idade (x) é dada pela equação: $y=5+(x-\mu) / s$, onde $\mathrm{x}=$ idade média, $\mu=$ média da distribuição hipotética de idades de desmame em uma dada população e $\mathrm{s}=$ desvio padrão. O modelo de regressão obtido pela análise de probitos apresenta o coeficiente de determinação $\mathrm{R}^{2}$, para a análise de ajustes de modelos.

Para analisar os determinantes da interrupção do aleitamento materno exclusivo em crianças menores de seis meses e interrupção do aleitamento materno em menores de um ano, efetuou-se regressão logística múltipla utilizando o procedimento stepwise forward selection, a um nível de significância de 5\%. As variáveis independentes estudadas foram: uso de chupeta, primiparidade, idade da mãe (menor de 20 anos ou 20 anos e mais), trabalho da mãe (dona de casa, trabalhadora formal, trabalhadora informal), escolaridade da mãe (até primeiro grau completo ou segundo grau e mais), procura por consulta médica (particular/convênio ou rede pública), tipo de parto (normal, fórceps ou cesárea), nascimento em "Hospital Amigo da Criança" (sim ou não), ajustadas pela idade da criança em dias e peso ao nascer.

Os dados foram digitados no aplicativo Amamunic, ${ }^{4}$ que forneceu relatórios sobre os indicadores de prevalência. Os intervalos de confiança (IC95\%) das prevalências foram calculados pelo programa CSAMPLE, do Epi-info, que considerou o delineamento amostral por conglomerados. Para o cálculo dos probitos e das medianas, foi usado o pacote estatístico SPSS v.7.0.

O projeto de pesquisa foi analisado e aprovado pelo Comitê de Ética em Pesquisa da Universidade Estadual de Londrina, sendo solicitado a todas as mães consentimento informado.

\section{Resultados}

Foram estudadas 2002 crianças menores de um ano, das quais $642(32,1 \%)$ eram menores de quatro meses e $988(49,3 \%)$ menores de seis meses.

A Tabela 1 apresenta a prevalência do Aleitamento Materno Exclusivo e do Aleitamento Materno Predominante, bem como seus intervalos de confiança (IC95\%) segundo a faixa etária das 
Tabela 1

Distribuição das crianças segundo a prevalência do aleitamento materno exclusivo (AME), do aleitamento materno predominante (AMP) e a faixa etária. Londrina, Paraná, 2002.

\begin{tabular}{lcc}
\hline Indicador & Prevalência (\%) & IC95\% \\
\hline AME $<4$ meses & 29,3 & $25,2-33,4$ \\
AME $<6$ meses & 21,0 & $18,2-23,9$ \\
AMP $<4$ meses & 20,7 & $17,2-24,2$ \\
AMP $<6$ meses & 16,5 & $13,7-19,3$ \\
\hline
\end{tabular}

\section{Figura 1}

Curva de probabilidade do aleitamento materno exclusivo (AME) em crianças menores de quaro meses. Londrina, Paraná, 2002.

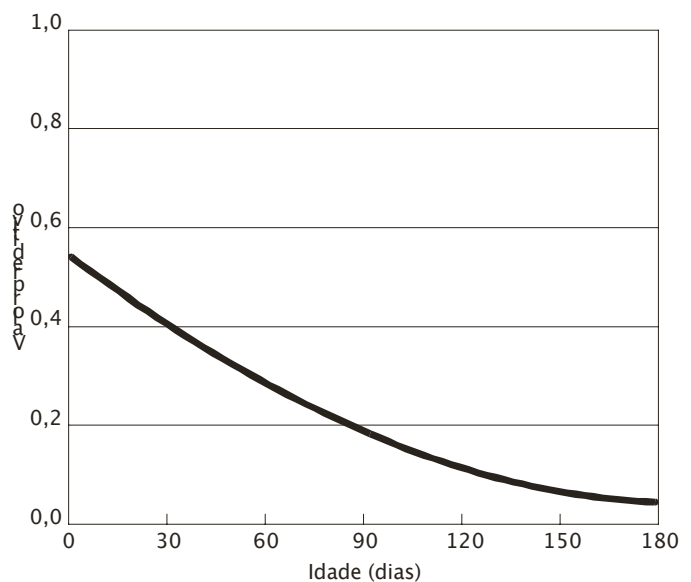

A Tabela 2 apresenta as variáveis que permaneceram no modelo final de regressão e que estão associadas significativamente ao AME em menores de seis meses. Verificou-se que após o ajuste pelas variáveis, idade em dias e peso ao nascer, apresentaram-se como determinantes para a interrupção do crianças. A prevalência do AM para crianças menores de um ano de idade foi de $62,40 \%$ com um IC95\%: 60,2-64,6.

Na Figura 1 observa-se a curva da probabilidade de aleitamento materno exclusivo em menores de quatro meses, com $\mathrm{R}^{2}=0,9527$. A mediana do AME é de 11,9 dias (IC95\%: 5,97-25,01).

A Figura 2 apresenta a curva da probabilidade do aleitamento materno, com o R2=0,9901. A mediana do AM foi 257,3 dias [IC95\%: 244,21-72,09].

A mediana do AM (257,3 dias) está próxima aos valores encontrados nos níveis nacionais. ${ }^{10}$

\section{Figura 2}

Curva de probabilidade do aleitamento materno (AM) em crianças menores de quatro meses. Londrina, Paraná, 2002.

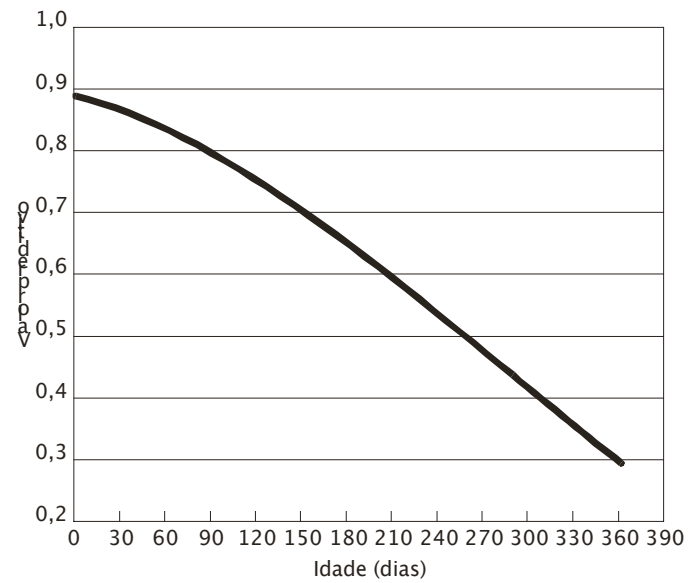

AME em menores de 180 dias as seguintes variáveis: uso de chupeta (OR=2,23; IC95\%: 1,43-3,47), primiparidade da mãe $(\mathrm{OR}=1,63$; IC95\%: 1,05-2,51) e o seguimento ambulatorial dessas crianças ocorrer na rede pública (OR=2,08; IC95\%: 1,26-3,43). 
Regressão logística dos fatores associados à interrupção do aleitamento materno exclusivo (AME) em menores de seis meses. Londrina, Paraná, 2002.

\begin{tabular}{|c|c|c|c|c|}
\hline Variável & $\mathbf{N}$ & AME (\%) & OR ajustado & IC(95\%) \\
\hline \multicolumn{5}{|l|}{ Uso de chupeta } \\
\hline Não & 506 & 28,5 & 1 & \\
\hline Sim & 478 & 13,4 & 2,23 & $1,43-3,47$ \\
\hline \multicolumn{5}{|l|}{ Primiparidade } \\
\hline Não & 387 & 26,3 & 1 & \\
\hline Sim & 456 & 18,6 & 1,63 & $1,05-2,51$ \\
\hline \multicolumn{5}{|l|}{ Consulta médica } \\
\hline Particular/Convênio & 215 & 25,1 & 1 & \\
\hline Rede pública & 756 & 19,7 & 2,08 & $1,26-3,43$ \\
\hline
\end{tabular}

A Tabela 3 mostra, para crianças menores de um ano, as variáveis que permaneceram no modelo final de regressão e que estão associadas significativamente ao AM em menores de um ano. Após o ajuste do modelo pelas variáveis, a idade das crianças em dias e peso ao nascer, destaca-se como determinante para a interrupção do AM o uso de chupeta, que apresenta um risco de 7,59 para o desmame em relação às crianças que não usam chupeta.

Tabela 3

Regressão logística dos fatores associados à interrupção do aleitamento materno (AM) em crianças menores de um ano. Londrina, Paraná, 2002.

\begin{tabular}{|c|c|c|c|c|}
\hline Variável & $\mathbf{N}$ & AM (\%) & OR ajustado & IC(95\%) \\
\hline \multicolumn{5}{|l|}{ Uso de chupeta } \\
\hline Não & 824 & 79,5 & 1 & \\
\hline Sim & 423 & 44,4 & 7,59 & $5,63-10,23$ \\
\hline \multicolumn{5}{|l|}{ Primiparidade } \\
\hline Não & 283 & 67,5 & 1 & \\
\hline Sim & 1357 & 65,1 & 1,78 & $1,19-2,65$ \\
\hline \multicolumn{5}{|l|}{ Consulta médica } \\
\hline Particular/Convênio & 868 & 70,0 & 1 & \\
\hline Rede pública & 760 & 60,1 & 1,72 & $1,28-2,33$ \\
\hline \multicolumn{5}{|l|}{ Trabalho da Mãe } \\
\hline Dona de casa & 757 & 70,4 & 1 & \\
\hline Trabalho formal & 265 & 54,7 & 1,61 & $1,10-2,35$ \\
\hline Trabalho informal & 259 & 66,0 & 1,19 & $0,81-1,73$ \\
\hline Desempregada & 126 & 54,8 & 1,23 & $0,73-2,0$ \\
\hline
\end{tabular}




\section{Discussão}

O inquérito para a avaliação das práticas alimentares de crianças menores de um ano de idade do município de Londrina em 2002, por ocasião da segunda etapa da "Campanha Nacional de Vacinação", mostrou ser de fácil aplicação tendo contribuido para fortalecr as parcerias entre as Universidades e a Secretaria Municipal de Saúde através do CALMA. A cobertura na campanha de crianças menores de um ano alcançou um percentual de $76,7 \%$ atingindo um nível adequado para a população do município.

Pesquisa semelhante a esta, realizada em municípios do Estado de São Paulo, mostra que o AME nos primeiros quatro meses de vida raramente alcançam índices superiores a 30\%. De 84 municípios estudados, 27 apresentaram prevalência de AME superior a $20 \%$, enquanto a prevalência do AMP, em menores de quatro meses, foi superior a $20 \%$ e alcançou 72 $(85,7 \%)$ municípios estudados. ${ }^{4}$ Situação semelhante foi encontrada em Londrina, onde a prevalência do AME em menores de quatro meses foi de $29,3 \%$ e do AMP foi de 20,7\%. Esses índices, depois de analisados pelos membros do CALMA, chamaram a atenção diante do esforço que Hospitais, Secretaria de Saúde e Universidades de Londrina vêm fazendo ao longo dos anos para implementar a prática do aleitamento materno.

Dentre esses esforços, cabe lembrar os cursos de manejo da amamentação, os cursos de aconselhamento realizados para profissionais dos hospitais e das unidades de saúde da rede pública do município, além da promoção da semana mundial de aleitamento materno, desde 1995. Entre 1995 a 1997, a Secretaria Municipal de Saúde lançou a iniciativa "Unidade Básica de Saúde Amiga da Criança", experiência que formalizou a importância do resgate da prática do AM pela rede básica de saúde no município de Londrina. 11 Lamentavelmente, mudanças administrativas levaram à interrupção do projeto após esse período. A cidade conta também com três hospitais "Amigos da Criança", um Banco de Leite Humano e uma Clínica Pública de Lactação.

Analisando a tendência ao longo do tempo em Londrina, o tempo mediano do AME foi de 11,9 dias, abaixo dos valores encontrados em Curitiba (34,6 dias), na região Sul do país $(39,1$ dias) e no Brasil que é de 23,4 dias. Porém, existem capitais onde as medianas encontradas foram mais baixas, como é o caso de Cuiabá (5,1 dias) e Maceió, com 8,2 dias. 10 Quanto à mediana do $\mathrm{AM}$, em Londrina encontrou-se 257,4 dias, valor um pouco abaixo da média nacional (295,9 dias) e acima dos valores encontrados em Curitiba (221,9 dias) e na região Sul do país, que é de 225,2 dias. 10 É importante salientar que esforços de equipes podem modificar esses índices, como mostra o trabalho de Vannuchi, pois através da implantação da "Iniciativa Hospital Amigo da Criança" (IHAC) em um hospital escola de Londrina, conseguiu-se elevar de 12 (1994) para 45 dias (1998) a prevalência do AME para crianças que nasceram e necessitaram permanecer internadas na Unidade de Terapia Intensiva desse hospital.12

A baixa mediana do AME e a alta mediana do AM em Londrina, sugerem a introdução precoce de alimentos líquidos não nutritivos, leites artificiais, mingaus e outros alimentos associados à amamentação, o que aumenta o risco de infecções, além do que, quando utilizados em mamadeiras, pode haver interferência na sucção do lactente, influenciando na freqüência das mamadas ao seio e comprometendo o AME. $5,12-14$

As variáveis associadas à interrupção do AME e do AM (uso de chupeta, paridade da mãe, idade materna, trabalho materno fora de casa e atendimento das crianças pela rede pública) encontradas em Londrina foram também evidenciadas em outros estudos. 5, 15-17

Um estudo realizado por Cotrim et al. 18 sobre o uso de chupeta e aleitamento materno, mostra que $61,3 \%$ das crianças menores de quatro meses haviam feito uso de chupeta nas últimas 24 horas. Em Londrina encontrou-se percentual menor (48,1\%), mostrando que as crianças que não usam chupeta possuem uma chance 2,23 vezes maior para o AME e de 7,59 vezes maior para o AM. Esses resultados estão de acordo com os achados de Soares et al. 19 que confirmam a associação entre o uso de chupeta e menor duração do AM e AME. Há necessidade de se intensificar a discussão com mães, profissionais de saúde e a sociedade sobre as desvantagens do uso de chupeta em releção ao AM. Quanto à paridade, detectou-se que as primíparas possuem um risco maior para a não utilização do $\mathrm{AME}$, resultado semelhante ao de Venancio, ${ }^{4}$ que observou que a chance de se prolongar o tempo do AM, eleva-se de acordo com o aumento do número de filhos, principalmente quando a mãe é bem sucedida na amamentação dos filhos mais velhos.

Observou-se, também, que as crianças que realizavam seguimento ambulatorial na rede pública, tiveram uma chance 2,08 vezes maior de interromper o AME do que aquelas acompanhadas na rede privada e conveniada. A menor prevalência de AME nas crianças atendidas pela rede pública provavelmente pode ser atribuída às piores condições socioeconômicas da população atendida pelo SUS.4,15 Esses 
achados mostram a necessidade de se reavaliar e reorganizar as ações pró-amamentação que estão sendo desenvolvidas na rede de saúde pública de Londrina.

Quando se analisou o trabalho materno, a mãe que trabalha fora de casa apresentou uma chance 1,61 vez maior para interromper o AM em crianças menores de um ano. Esses resultados diferem dos achados de Vezozzo 20 que, em pesquisa realizada em Londrina, mostrou que a prática do AM entre as mulheres trabalhadoras estudadas estava bastante difundida, pois $97 \%$ delas iniciaram a amamentação e a duração mediana encontrada foi de 90 dias para o AME e 180 dias para o AM. Entretanto esse autor estudou somente trabalhadoras com registro em carteira. Há necessidade de se ampliar e intensificar a discussão sobre os mecanismos de proteção do AM para as mães trabalhadoras no mercado informal.

\section{Conclusões}

Embora Londrina venha desenvolvendo atividades relativas ao AM há décadas e atualmente conte com três "Hospitais Amigos da Criança, os resultados deste trabalho revelaram uma prevalência e uma mediana do AME muito aquém da proposta pela O rganização Mundial da Saúde (OMS), mostrando a necessidade de uma reavaliação e reorganização das políticas dos serviços de saúde do município, no sentido de se garantir maior envolvimento de profissionais de saúde e áreas afins, no estímulo e aconselhamento ao aleitamento materno. Muitos esforços estão sendo desenvolvidos no Brasil para a implantação de políticas de incentivo e apoio ao aleitamento materno. Inquéritos como este são importantes instrumentos de acompanhamento e avaliação dessas políticas, tendo em vista o alcance da meta proposta pela OMS, de que $100 \%$ das crianças até os seis meses de vida recebam apenas o leite materno como alimento. Os dados desta pesquisa servirão para redirecionar o trabalho desenvolvido pelo CALMA no sentido de buscar estratégias para aumentar a prevalência do AME e AM em Londrina.

\section{Referências}

1. WHO (World Health Organization). Global strategy for infant and young child feeding. Geneva; 2001. (Document A54/7).

2. Londrina. Prefeitura. Decreto Lei n. ${ }^{\circ} 245$ : ins titui o Comitê de Estímulo ao Aleitamento Materno no Município de Londrina (CALMA). Folha de Londrina, 6 de maio de 1995.

3. Venancio SY, Escuder MML, Kitoko P, Rea MF, Monteiro CA. Freqüência e determinantes do aleitamento materno em municípios do Estado de São Paulo. Rev Saúde Pública 2002; 36: 313-8.

4. Venancio SY. Determinantes individuais e contextuais do aleitamento materno exclusivo nos primeiros seis meses de vida em cento e onze municípios do Estado de São Paulo [tese doutorado]. São Paulo: Faculdade de Saúde Pública da Universidade de São Paulo; 2002.

5. Audi CAF, Corrêa MAS, Latorre MRDO. Alimentos complementares e fatores associados ao aleitamento materno e ao aleitamento materno exclusivo em lactentes até 12 meses de vida em Itapira, São Paulo, 1999. Rev Bras Saúde Matern Infant 2003; 3: 85-93.
6. IBGE (Instituto Brasileiro de Geografia e Estatística). Cidades@. Disponível em: <http://www.ibge.gov.br/ cidadesat/default.php. [2005 jan 12].

7. Silva NN. Amostragem probabilística: um curso introdutório. São Paulo: EDUSP; 1998.

8. WHO (World Health Organization). Indicators for assessing breastfeeding pratices. Update. Programme Control Diarrhoeal Dis 1991; (10):1-4.

9. Finney, DJ. Probit analysis. 3rd ed. Cambridge: Cambridge University Press; 1980.

10. Ministério da Saúde. Pesquisa de prevalência do aleitamento materno nas capitais e no Distrito Federal. Brasília (DF); 2001.

11. Prando C, Poli LMC. Relato de experiência: implantação do projeto Iniciativa Unidade Básica de Saúde Amiga da Criança de Londrina. Anais do V Encontro Nacional de Aleitamento Materno; 1997 set. 17-21; Londrina; Brasil; 1997. Londrina: CALMA; 1997.

12. Vannuchi MTO. Implantação e avaliação da Iniciativa Hospital Amigo da Criança na Unidade de Neonatologia do Hospital Universitário Regional do Norte do Paraná, 
Londrina [tese doutorado]. São Paulo: Faculdade de Saúde Pública da Universidade de São Paulo; 2002.

13. OMS (Organização Mundial da Saúde). Evidências científicas dos dez passos para o sucesso no aleitam ento materno. Brasília (DF); 2001.

14. Santiago LB, Bettiol H, Barbieri MA, Gutierrez MRP, Del Ciampo LA. Incentivo ao aleitamento materno: a importância do pediatra com treinamento específico. J Pediatr (Rio J) 2003; 79: 504- 12.

15. Lima TM, Osório MM. Perfil e fatores associados ao aleitamento materno em crianças menores de 25 meses da Região Nordeste do Brasil. Rev Bras Saúde Matern Infant 2003; 3 : 305-14.

16. Victora CG, Behague DP, Barros FC, Olinto MTA, Weiderpass E. Pacifiers'use and short breastfeeding duration: cause, consequence or coincidences? Pediatrics 1997; 99: 445-53.
17. Victora CG, Olinto MT, Barros, FC. Use of pacifiers and breastfeeding duration. Lancet 1993; 341: 404-6.

18. Cotrim LC, Venancio SY, Escuder MML. Uso de chupeta e amamentação em crianças menores de quatro meses no estado de São Paulo. Rev Bras Saúde Infant 2002; 2: 253-61.

19. Soares MEM, Giugliani ERJ, Braun ML, Salgado ACN, Oliveira AP, Aguiar PR. Uso de chupeta e sua relação precoce em população de crianças nascidas em hospital amigo da criança. J Pediatr (Rio J) 2003; 79: 309-16.

20. Vezozzo KMK. Amamentação e trabalho e feminino: a contribuição das leis e das empresas. [dissertação mestrado]. Londrina: Centro de Ciências da Saúde da Universidade Estadual de Londrina; 2000.

Recebido em 7 de julho de 2004

Versão final em 19 de abril de 2005

Aprovado em 6 de maio de 2005 